\title{
COMPLEX NONOSCILLATION THEOREMS AND CRITERIA OF UNIVALENCE $\left.{ }^{1}\right)$
}

\author{
BY \\ BINYAMIN SCHWARZ
}

1. Introduction. In this paper we shall consider functions $p(z)$ regular in the open unit circle $E$ and the solutions $y(z)$ of the differential equation

$$
y^{\prime \prime}(z)+p(z) y(z)=0 \text {. }
$$

The ratio

$$
f(z)=u(z) / v(z)
$$

of any two independent solutions $u(z)$ and $v(z)$ of (1.1) will be a function $f(z)$, meromorphic in $E$ with only simple poles, and such that $f^{\prime}(z) \neq 0$. We shall say that a meromorphic function which satisfies these two conditions belongs to the restricted class. The Schwarzian derivative of $f(z)$,

$$
\{f(z), z\}=\left[f^{\prime \prime}(z) / f^{\prime}(z)\right]^{\prime}-\left[f^{\prime \prime}(z) / f^{\prime}(z)\right]^{2} / 2
$$

is connected with $p(z)$ by

$$
\{f(z), z\}=2 p(z) .
$$

Conversely, for any meromorphic functions of the restricted class the Schwarzian derivative is regular.

The vanishing of a (nontrivial) solution

$$
y(z)=A u(z)+B v(z)
$$

of (1.1) at the points $z_{1}, \cdots, z_{n}$ is equivalent to $f(z)$ assuming at these points the value $-B A^{-1}$. It follows that $f(z)$ is univalent in $E$ if no solution of (1.1) (except the solution $y(z) \equiv 0$ ) has more than one zero in $E$. Conversely, every univalent function $f(z)$ in $E$ can be written as the ratio of two independent solutions of the equation (1.1) where $p(z)$ is defined by (1.4). These connections were first stated by $Z$. Nehari in $[6]\left({ }^{2}\right)$ and used there to obtain the following theorem (Theorem I, [6]): that

In order that the analytic function $f(z)$ be univalent in $|z|<1$ it is necessary

Received by the editors July 1, 1954.

(1) This paper represents part of a thesis submitted to the Graduate School of Washington University in partial fulfillment of the requirements for the degree of Doctor of Philosophy. The author wishes to thank Professor Zeev Nehari for his generous help in the preparation of this paper.

(3) Square brackets refer to the bibliography at the end of the paper. 


$$
|\{f(z), z\}| \leqq 6 /\left(1-|z|^{2}\right)^{2}
$$

and sufficient that

$$
|\{f(z), z\}| \leqq 2 /\left(1-|z|^{2}\right)^{2} \text {. }
$$

This theorem is sharp, as is shown for the necessary condition by the Koebe extremal function $f(z)=z /(1-z)^{2}$, and for the sufficient condition by an example due to E. Hille [4]; we shall discuss this example in detail later.

2. A nonoscillation theorem for the unit circle. The differential equation (1.1) is called nonoscillatory in $E$ if none of its solutions (except $y(z) \equiv 0)$ has infinitely many zeros in $E$. Correspondingly we call a single-valued meromorphic function finitely-valent in a domain $D$ if the equation $f(z)=a$ has for each $a$ only a finite,number of solutions $z$ in $D$. By a slight modification of the proof leading to the sufficient condition (1.6) of Theorem I in [6], we obtain now the following nonoscillation theorem:

THEOREM 1. Let $p(z)$ be regular in $|z|<1$ and assume there exists $x_{0}$, $0<x_{0}<1$, such that for all $z$ with $x_{0}<|z|<1$

$$
|p(z)| \leqq 1 /\left(1-|z|^{2}\right)^{2} .
$$

Then the differential equation

$$
y^{\prime \prime}(z)+p(z) y(z)=0
$$

is nonoscillatory in $|z|<1$. Moreover, for every $\gamma>0$, there exists a function $q(z)$, regular in $|z|<1$, such that for all $z$ in $|z|<1$

$$
|q(z)| \leqq\left(1+4 \gamma^{2}\right) /\left(1-|z|^{2}\right)^{2}
$$

and such that the equation

$$
v^{\prime \prime}(z)+q(z) v(z)=0
$$

is oscillatory in $|z|<1$ (i.e. has at least one solution $v(z)$ with an infinity of zeros there).

Proof. Let $z_{1}, z_{2}\left(z_{1} \neq z_{2}\right)$ be any two points inside the open unit circle $E . z_{1}, z_{2}$ determine uniquely a circle $C$ passing through them and orthogonal to $|z|=1$. Let us call that part of $C$ which lies between $z_{1}$ and $z_{2}$ and inside $E$ "the orthogonal arc between $z_{1}$ and $z_{2}{ }^{\prime}$ and let us denote it by $\left[z_{1} z_{2}\right]$. Let $x_{0}$ be fixed and denote the ring $x_{0}<|z|<1$ by $R$. Assume now that there exists a nontrivial solution $y(z)$ of (1.1) with infinitely many zeros in $E$. From this infinity of zeros we choose a sequence converging to a point $\alpha$ on $|z|=1$. By an elementary geometric consideration it follows that we can choose two zeros $z_{1}$ and $z_{2}$ of $y(z)$, belonging to this sequence, such that they, together with the orthogonal arc between them, lie in $R$.

There exists a linear transformation from $|z|<1$ onto $|\zeta|<1$ given by 


$$
z=e^{i \theta \cdot \frac{\zeta-\alpha}{1-\bar{\alpha} \zeta}}
$$

which carries $z_{1}$ and $z_{2}$ into $\zeta=\rho$ and $\zeta=-\rho$ respectively $(0<\rho<1)$. (2.3) transforms $\left[z_{1} z_{2}\right]$ into the segment $(-\rho, \rho)$. Let $f(z)$ be the ratio of any two independent solutions of (1.1) and define $g(\zeta)$ for $|\zeta|<1$ by

$$
g(\zeta)=f\left(e^{i \theta} \frac{\zeta-\alpha}{1-\bar{\alpha} \zeta}\right) .
$$

The substitution (2.3) transforms the differential equation (1.1) into

$$
y_{1}^{\prime \prime}(\zeta)+p_{1}(\zeta) y_{1}(\zeta)=0
$$

where

$$
2 p_{1}(\zeta)=\{g(\zeta), \zeta\}
$$

and

$$
y\left(e^{i \theta} \frac{\zeta-\alpha}{1-\bar{\alpha} \zeta}\right)=y_{1}(\zeta) \sigma(\zeta) .
$$

Here $\sigma(\zeta)$ is regular and $\neq 0$ in $|\zeta|<1$. It follows that there exists a solution $y_{1}(\zeta) \not \equiv$ of $(2.5)$ such that $y_{1}(\rho)=y_{1}(-\rho)=0$. Setting $\zeta=x+i y$, multiplying (2.5) on the segment $(-\rho, \rho)$ by $\bar{y}_{1} d x$, and integrating from - $\rho$ to $\rho$, we obtain

$$
\int_{-\rho}^{\rho}\left|y_{1}^{\prime}\right|^{2} d x=\int_{-\rho}^{\rho} p_{1}\left|y_{1}\right|^{2} d x .
$$

Writing $y_{1}=u+i v$ we have

$$
\int_{-\infty}^{p}\left(u_{x}^{2}+v_{x}^{2}\right) d x=\int_{\rightarrow}^{p} p_{1}\left(\dot{u}^{2}+v^{2}\right) d x .
$$

It can be shown that (2.3) and (2.4) imply

$$
|\{f(z), z\}|\left(1-|z|^{2}\right)^{2}=|\{g(\zeta), \zeta\}|\left(1-|\zeta|^{2}\right)^{2} .
$$

It follows therefore by (2.1), (1.4) and (2.6) that

$$
\left|p_{1}(x)\right| \leqq \frac{1}{\left(1-x^{2}\right)^{2}}, \quad-\rho \leqq x \leqq \rho .
$$

Hence,

$$
\left|\int_{-\rho}^{\rho} p_{1}\left(u^{2}+v^{2}\right) d x\right| \leqq \int_{-\rho}^{\rho} \frac{u^{2}+v^{2}}{\left(1-x^{2}\right)^{2}} d x<\rho^{2} \int_{-\rho}^{\rho} \frac{u^{2}+v^{2}}{\left(\rho^{2}-x^{2}\right)^{2}} d x .
$$

Now the integral inequality 


$$
\rho^{2} \int_{-\rho}^{\rho} \frac{u^{2} d x}{\left(\rho^{2}-x^{2}\right)^{2}}<\int_{-\rho}^{\rho} u^{\prime 2} d x
$$

holds for continuously differentiable real functions $u(x),-\rho \leqq x \leqq \rho$, which have at $\pm \rho$ zeros of the first order [6]. We may apply this inequality to both $u$ and $v$ and obtain, in view of (2.10),

$$
\left|\int_{-\rho}^{\rho} p_{1}\left(u^{2}+v^{2}\right) d x\right|<\int_{-\rho}^{\rho}\left(u_{x}^{2}+v_{x}^{2}\right) d x,
$$

which gives the desired contradiction to (2.8) and we have therefore proved the main assertion of the theorem.

To prove the sharpness, consider for any $\gamma>0$ and any complex $C$ the function

$$
v(z)=\left(z^{2}-1\right)^{1 / 2} \sin \{\gamma \log (1+z) /(1-z)-C\} .
$$

$v(z)$ is regular for $|z|<1$; to determine $v(z)$ uniquely, take in its definition the principal branch for the square root and for the logarithm. The function

$$
q(z)=-v^{\prime \prime}(z) / v(z)=\left(1+4 \gamma^{2}\right) /\left(1-z^{2}\right)^{2}
$$

is then regular in $E$ and satisfies condition (2.2). It is easily seen that if $|\operatorname{Im}\{C\}|<\pi \gamma / 2$ (in particular if $C$ is real) then $v(z)$ has infinitely many zeros in $E$ and so the proof of Theorem 1 is concluded.

The differential equation considered just now is a special case of Hille's example, mentioned above. Hille considers the equation

$$
y^{\prime \prime}(z)+\frac{a}{\left(1-z^{2}\right)^{2}} y(z)=0, \quad|z|<1,
$$

where $a$ is the complex parameter. His result is that no solution of (2.12) assumes more than one zero in $E$ if, and only if, $a$ belongs to the interior or to the boundary of the cardioid given by $a=-2 e^{i \phi}-e^{2 i \phi}$. This cardioid goes through the points $a=+1$ and $a=-3$, contains $|a| \leqq 1$, and is contained in $|a| \leqq 3$; the point $a=-3$ gives the differential equation corresponding to the Koebe extremal function, while $a=1+4 \gamma^{2}(\gamma>0)$ shows the sharpness of the sufficiency condition of Theorem I of [6] and, as just shown, also the sharpness of Theorem 1.

This nonoscillation theorem may now be stated as a criterion of finitevalence for meromorphic functions of the restricted class and, in fact, for meromorphic functions in general.

COROLlaRy 1. Let $f(z)$ be meromorphic in $|z|<1$ and assume that

$$
|\{f(z), z\}| \leqq 2 /\left(1-|z|^{2}\right)^{2} \text { for } x_{0}<|z|<1, \quad 0<x_{0}<1 .
$$

Then $f(z)$ is finitely-valent in $|z|<1$. 
Proof. Assume that there exists a complex number $a$ (which may be $\infty$ ) such that $f(z)-a=0$ has an infinity of roots in $E$. It follows then again that there exist $z_{1}, z_{2},\left(z_{1} \neq z_{2}\right)$ such that $f\left(z_{1}\right)=f\left(z_{2}\right)=a$, and that $z_{1}, z_{2}$ and the orthogonal arc between them lie in $R$. Consider now $f(z)$ and the corresponding differential equation $y^{\prime \prime}(z)+p(z) y(z)=0$ not in $E$, but only in any simplyconnected domain $D$ containing the arc $\left[z_{1} z_{2}\right]$ and contained in $R$. We obtain therefore a solution $y(z)$ of (1.1), analytic (and therefore single-valued) in $D$, such that $y\left(z_{1}\right)=y\left(z_{2}\right)=0$, while $p(z)$ satisfies (2.1) in $D$ (and especially on $\left.\left[z_{1} z_{2}\right]\right)$. But only this was used in the proof of Theorem 1 ; the behavior of $p(z)$ and $y(z)$ outside $D$ (and of $p_{1}(\zeta)$ and $y_{1}(\zeta)$ outside the map of $D$ under (2.3)) is obviously immaterial for the proof.

The sharpness of Theorem 1 implies the sharpness of the corollary. By (2.11) and (1.2) this is established by the functions

$$
f(z)=\tan \left(\gamma \log \frac{1+z}{1-z}\right), \quad \gamma>0 .
$$

In a recent paper [7], Z. Nehari obtained a series of nonoscillation theorems for the equation $y^{\prime \prime}(z)+p(z) y(z)=0$ in various domains. For the unit circle he proved (Theorem III, [7]) that if $p(z)$ is regular in $|z|<1$ and if

$$
\int_{0}^{2 \pi}\left|p\left(e^{i \theta}\right)\right| d \theta<\infty
$$

then the differential equation is nonoscillatory. The integral on the left-hand side of (2.14) is defined as the limit, for $\rho \rightarrow 1$, of the nondecreasing function

$$
\int_{0}^{2 \pi}\left|p\left(\rho e^{i \theta}\right)\right| d \theta
$$

and (2.14) is therefore equivalent to

$$
\int_{0}^{2 \pi}\left|p\left(\rho e^{i \theta}\right)\right| d \theta<C, \quad C<\infty, 0<\rho<1 .
$$

This theorem may be deduced from Theorem 1. Indeed, setting

$$
p(z)=\sum_{n=0}^{\infty} a_{n} z^{n}
$$

(2.15) implies

$$
\left|a_{n}\right| \leqq \frac{1}{2 \pi} \int_{0}^{2 \pi} \frac{\left|p\left(\rho e^{i \theta}\right)\right|}{\rho^{n+1}} \rho d \theta<\frac{C}{2 \pi \rho^{n}}, \quad n=0,1, \cdots .
$$

Letting $\rho \rightarrow 1$, we obtain $\left|a_{n}\right| \leqq C / 2 \pi=C_{1}$ and therefore 


$$
|p(z)| \leqq \sum_{n=0}^{\infty}\left|a_{n}\right||z|^{n} \leqq \frac{C_{1}}{1-|z|} .
$$

This implies now the existence of an $x_{\theta}$, such that (2.1) holds for $x_{0}<|z|<1$, i.e., the assumption of Theorem 1 is satisfied.

3. A finite-valence theorem for multiply-connected domains. Let $D$ be a simply-connected domain in the $z$-plane, having at least two boundary points and let $w=\psi(z)$ be a function mapping $D$ onto $|w|<1$. Let $D^{\prime}$ be any closed domain lying in the interior of $D$ and denote by $R^{\prime}$ the domain $D-D^{\prime}$. The map of $R^{\prime}$ under the transformation $w=\psi(z)$ covers a circular ring $R, x_{0}<|w|$ $<1$, with $0<x_{0}<1$ and $x_{0}$ near enough to 1 . Let $f(z)$ be a meromorphic function in $D$ and define $g(w)$ in $|w|<1$ by

$$
g(w)=f\left(\psi^{-1}(w)\right) .
$$

$f(z)$ is finitely-valent in $D$ if and only if $g(w)$ is so in $|w|<1$. The transformation formula for $\{f(z), z\}$ under the conformal mapping $w=\psi(z)$ is

$$
\{g(w), w\}=\left(\frac{d z}{d w}\right)^{2}[\{f(z), z\}-\{w, z\}] .
$$

Applying now Corollary 1 to $g(w)$ it follows that $f(z)$ will be finitely-valent in $D$ if the condition

$$
|\{f(z), z\}-\{\psi(z), z\}| \leqq \frac{2}{\left(1-|\psi(z)|^{2}\right)^{2}}\left|\frac{d \psi}{d z}\right|^{2}
$$

holds for all $z$ in $D-D^{\prime}$. Similarly it follows that if $p(z)$ is regular in $D$ and if

$$
\left|p(z)-\frac{1}{2}\{\psi(z), z\}\right| \leqq \frac{1}{\left(1-|\psi(z)|^{2}\right)^{2}}\left|\frac{d \psi}{d z}\right|^{2}
$$

holds for all $z$ in $D-D^{\prime}$ then the differential equation (1.1) is nonoscillatory in $D$.

We remark that conditions (3.3) and (3.4) are independent of the normalization of the Riemann mapping function $w=\psi(z)$ mapping $D$ onto $|w|<1$. Let $w_{1}=\psi_{1}(z)$ be another such function mapping $D$ onto $\left|w_{1}\right|<1$. The function $w_{1}(w)=\psi_{1}\left(\psi^{-1}(w)\right)$ is a linear mapping of $|w|<1$ onto $\left|w_{1}\right|<1$ and it follows, by the invariance of the Schwarzian derivative with respect to all linear transformations, that $\left\{w_{1}(z), z\right\}=\{w(z), z\}$, i.e.,

$$
\left\{\psi_{1}(z), z\right\}=\{\psi(z), z\} \text {. }
$$

Moreover, for a linear mapping of the unit circle onto itself, the relation

$$
\frac{1-\left|w_{1}(w)\right|^{2}}{1-|w|^{2}}=\left|\frac{d w_{1}}{d w}\right|
$$

holds, which implies 


$$
\frac{1}{\left(1-\left|\psi_{1}(z)\right|^{2}\right)^{2}}\left|\frac{d \psi_{1}}{d z}\right|^{2}=\frac{1}{\left(1-|\psi(z)|^{2}\right)^{2}}\left|\frac{d \psi}{d z}\right|^{2} .
$$

(3.5) and (3.6) show clearly that conditions (3.3) and (3.4) are independent of the normalization of the mapping function $\psi(z)$.

As an example for condition (3.4) we mention the half-plane $\operatorname{Re}\{z\}>0$. In this case,

$$
w=\psi(z)=\frac{z-1}{z+1}
$$

and condition (3.4) becomes

$$
|p(z)| \leqq 1 / 4 x^{2} \quad(z=x+i y) .
$$

The differential equation (1.1) is therefore nonoscillatory in $\operatorname{Re}\{z\}>0$ if (3.7) holds for all points of this half-plane except those belonging to a closed bounded subset. Sharpness is shown by the Euler equation

$$
y^{\prime \prime}(z)+\frac{C}{4 z^{2}} y(z)=0 \quad(C>1),
$$

which plays an all-important role in the real nonoscillation theory (see Hille [3]).

In the case of the strip $|\operatorname{Im}\{z\}|<\pi / 4$, we have

$$
w=\psi(z)=\tan (i z)
$$

and (3.4) becomes

$$
|p(z)+1| \leqq 1 /(\cos 2 y)^{2} \quad(z=x+i y) .
$$

While condition (3.3) was stated for schlicht simply-connected domains, it will evidently also hold for non-schlicht domains which can be mapped onto the unit circle. This is the case for the universal covering surfaces of multiplyconnected domains with more than two boundary points. Restricting ourselves to domains bounded by a finite number of Jordan curves $\left({ }^{3}\right)$, we obtain

LeMma 1. Let $D$ be a multiply-connected domain in the z-plane, bounded by a finite number of Jordan curves. Let $S$ be its universal covering surface (and denote the affix of $S$ also by $z$ ). Let $w=\psi(z)$ map $S$ onto $|w|<1$ and let $D^{\prime}$ be any closed domain inside $D$. A function $f(z)$, meromorphic and single-valued in $D$, will be finitely-valent there, if condition (3.3) holds for all $z$ in $D-D^{\prime}$.

We remark first that the expressions $\{\psi(z), z\}$ and

$$
\frac{2}{\left(1-|\psi(z)|^{2}\right)^{2}}\left|\frac{d \psi}{d z}\right|^{2}
$$

(3) See Bieberbach [1, pp. 44-56] and Nevanlinna [9, pp. 21, 22]. 
are not only, as above, independent of the normalization of $\psi(z)$, i.e., singlevalued as functions on $S$, but they are, in addition, single-valued functions in $D$. This follows from the fact that the different branches of $\psi(z)$ (as a function in $D$ ) correspond to linear transformations of $|w|<1$ onto itself.

For the proof of the lemma, let $z$ be the coordinate in $D$ and not on $S$, so that $\psi(z)$ is a many-valued function. By connecting the $n$ boundary curves $C_{1}, \cdots, C_{n}$ of $D$ by $n-1$ cuts $\gamma_{1}, \cdots, \gamma_{n-1}$, we obtain a simply-connected domain $D^{*}$. $D^{*}$ allows us to fix uniquely a branch $\psi_{i}(z)$ of $\psi(z)$ (which will be regular and single-valued for $\left.z \in D^{*}\right) . \psi_{i}(z)$ maps $D^{*}$ onto a simply-connected domain $F_{i}^{*}$ whose boundary consists of $2 n-2 \operatorname{arcs} C_{1}^{i}, \cdots, C_{2 n-2}^{i}$ on $|w|=1$ which are the maps of the $2 n-2$ components into which the curves $C_{1}, \cdots, C_{n}$ are decomposed by the system of cuts, and of $2 n-2$ arcs inside $|w|=1$ which are the maps of the $n-1$ cuts $\gamma_{1}, \cdots, \gamma_{n-1}$. We adjoin $n-1$ of these arcs, called $\gamma_{1}^{i}, \cdots, \gamma_{n-1}^{i}$, to $F_{i}^{*}$ and obtain a fundamental domain $F_{i}$. To each $z \in D$ corresponds exactly one $w=\psi_{i}(z)$ in $F_{i}$. Let us call the arcs $\gamma_{1}^{i}, \cdots, \gamma_{n-1}^{i}$, together with the $n-1$ equivalent arcs which belonged to the boundary of $F_{i}^{*}$, the inner boundaries of $F_{i} . \psi_{i}(z)$ maps $D^{\prime}$ onto a region $F_{i}^{\prime}$. While certain segments of the inner boundaries of $F_{i}$ belong to $F_{i}^{\prime}$ or to its boundary, it is clear that $F_{i}^{\prime}$ lies inside a circle $|w| \leqq x_{0}<1$.

Let now the function $g(w)=f\left(\psi^{-1}(w)\right)$ be defined only for $w \in F_{i}$. This function is meromorphic and single-valued, and it follows that the condition

$$
|\{g(w), w\}| \leqq 2 /\left(1-|w|^{2}\right)^{2}
$$

holds for $w \in F_{i} \cap R$ (where $R$ is again the ring $x_{0}<|w|<1$ ). This ensures the finite-valence of $g(w)$ in $F_{i}$ and therefore of $f(z)$ in $D$. Lemma 1 is established.

This lemma enables us now to obtain a simpler criterion for the finitevalence of single-valued meromorphic functions in the case in which the $n$ boundaries of the domain are analytic Jordan curves.

THEOREM 2. Let $D$ be a domain in the z-plane such that its boundary $C$ consists of a finite number of analytic Jordan curves. Let $z_{0} \in D$ and denote by $C_{\epsilon}$ the level curve $g\left(z, z_{0}, D\right)=\epsilon, \epsilon>0$, of the harmonic Green's function $g\left(z, z_{0}, D\right)$ of the domain $D$ with pole at $z_{0}$. Let $f(z)$ be meromorphic and single-valued in $D$ and set

$$
M(\epsilon)=\underset{z \in C_{\epsilon}}{\operatorname{Max}}|\{f(z), z\}| .
$$

If

$$
\lim _{\epsilon \rightarrow 0} \epsilon^{2} M(\epsilon)=0
$$

then $f(z)$ is finitely-valent in $D$.

Proof. Suppose $D$ is not simply-connected. Choose $\psi(z)$ on $S$ so that $\psi^{-1}(0)=z_{0}$. Define the different branches of $\psi(z)$ (as a function in $D$ ) as in the proof of Lemma 1, but assume that none of the cuts $\gamma_{1}, \cdots, \gamma_{n-1}$ go through 
$z_{0}$. Let the branch $\psi_{i}(z)$ be defined by $\psi_{i}\left(z_{0}\right)=0$, and consider the behavior of this branch in $D$ and on $C$. From the analyticity of the boundary curves it follows that $\psi_{i}(z)$ and its derivatives are piecewise analytic on $C$. Moreover, $d \psi_{i} / d z \neq 0$ in $\bar{D}=D \cup C$ and it follows that, for all $z$ in $D$,

$$
\left|\left\{\psi_{i}(z), z\right\}\right|=|\{\psi(z), z\}| \leqq M, \quad 0<M<\infty,
$$

and

$$
\left|d \psi_{i} / d z\right| \geqq m, \quad 0<m<\infty .
$$

For every $\epsilon>0$ let us now consider the following two closed regions in $D$ :

$$
D_{1}(\epsilon)=\left\{z: g\left(z, z_{0}, D\right) \geqq \epsilon\right\}
$$

and

$$
D_{2}(\epsilon)=\left\{z: z=\psi^{-1}(w),|w| \leqq e^{-\epsilon}\right\}
$$

We then have( $\left.{ }^{4}\right)$

$$
D_{1}(\epsilon) \supset D_{2}(\epsilon) \text {. }
$$

To prove this, we consider the Green's function $G\left(z, z_{0}, S\right)$ of the universal covering surface $S . z$ and $z_{0}$ are now both on $S$, the pole $z_{0}$ is defined by $\psi\left(z_{0}\right)=0$; i.e., $z_{0}$ is the point in the $i$ th sheet of $S$ whose trace in $D$ was also denoted by $z_{0}$. Let now $D_{2}^{*}(\epsilon)$ be the region on $S$ defined by

$$
D_{2}^{*}(\epsilon)=\left\{z: G\left(z, z_{0}, S\right) \geqq \epsilon\right\}, \quad z \in S .
$$

$D_{2}(\epsilon)$ is therefore the trace of $D_{2}^{*}(\epsilon) . g\left(z, z_{0}, D\right)$ was defined in $D$; we now associate with every $z \in S$ the value which $g\left(z, z_{0}, D\right)$ assumes at the trace of this point and so obtain a function $G\left(z, z_{0}, D\right) . G\left(z, z_{0}, D\right)$ is positive and harmonic on $S$, except for infinitely many logarithmic poles (at all points of $S$ whose trace in $D$ is $\left.z_{0}\right)$. Let us denote by $D_{1}^{*}(\epsilon)$ the union of the infinitely many replicas of $D_{1}(\epsilon)$ on $S$; i.e.,

$$
D_{1}^{*}(\epsilon)=\left\{z: G\left(z, z_{0}, D\right) \geqq \epsilon\right\},
$$$$
z \in S \text {. }
$$

By the just mentioned properties of $G\left(z, z_{0}, D\right)$ and the fact that $G\left(z, z_{0}, S\right)$ has only one pole (which coincides with one of the poles of $G\left(z, z_{0}, D\right)$ ) and vanishes at the boundary of $S$, it follows that for each $z \in S$

$$
G\left(z, z_{0}, D\right) \geqq G\left(z, z_{0}, S\right), \quad z \in S,
$$

and therefore

$$
D_{1}^{*}(\epsilon) \supset D_{2}^{*}(\epsilon),
$$$$
\epsilon>0 \text {. }
$$

Projecting now onto the $z$-plane, we obtain the desired relation (3.12).

(4) Nevanlinna [9, pp. 50-51]. 
(3.12) implies now that $|\psi(z)| \geqq e^{-\epsilon}$ for the level curve $C_{\epsilon}\left(g\left(z, z_{0}, D\right)=\epsilon\right)$ and in particular

$$
\left|\psi_{i}(z)\right| \geqq e^{-\epsilon}, \quad z \in C_{\epsilon}, \epsilon>0 .
$$

We have therefore for each $z \in C_{\epsilon}$

$$
1-\left|\psi_{i}(z)\right|^{2}=\left(1+\left|\psi_{i}(z)\right|\right)\left(1-\left|\psi_{i}(z)\right|\right)<2\left(1-e^{-s}\right)<2 \epsilon,
$$

which implies

$$
M(\epsilon)\left(1-\left|\psi_{i}(z)\right|^{2}\right)^{2}<4 M(\epsilon) \epsilon^{2} .
$$

Using now our assumption (3.9), it follows from (3.10), (3.11), and (3.13) that there exists $\epsilon_{0}>0$ so that

$$
\left|\{f(z), z\}-\left\{\psi_{i}(z), z\right\}\right| \leqq \frac{2}{\left(1-\left|\psi_{i}(z)\right|^{2}\right)^{2}}\left|\frac{d \psi_{i}}{d z}\right|^{2}
$$

for all $z$ with $0<g\left(z, z_{0}, D\right)<\epsilon_{0}$, i.e., for all $z \in D-D_{1}\left(\epsilon_{0}\right)$. In the last inequality the subscript indicating the branch is now unnecessary; the assumption of Lemma 1 is therefore fulfilled and we have proved the theorem for a multiply-connected domain.

If $D$ is simply-connected we use condition (3.3) (as stated at the beginning of this section). Relations (3.10) and (3.11) hold now for the single-valued function $\psi(z)$ and in this case, clearly, $D_{1}(\epsilon)=D_{2}(\epsilon)$. Theorem 2 is therefore established.

Using the theorem quoted at the end of $\S 2, Z$. Nehari proved the following theorem (Theorem IV, [7]):

If $p(z)$ is regular in a (simply-connected) domain $D$ bounded by an analytic Jordan curve $C$, and

$$
\int_{C}|p(z) d z|<\infty
$$

then the equation (1.1) is nonoscillatory in D.

The integral in (3.14) is defined as

$$
\lim _{\boldsymbol{\epsilon} \rightarrow 0} I(\boldsymbol{\epsilon}), \quad I(\boldsymbol{\epsilon})=\int_{C_{\epsilon}}|p(z) d z|, \quad \quad \epsilon>0,
$$

where $C_{\epsilon}$ is again the level curve of $g\left(z, z_{0}, D\right) . I(\epsilon)$ grows monotonically with decreasing $\epsilon$ and (3.14) is therefore equivalent to

$$
\int_{C_{\epsilon}}|p(z) d z|<M, \quad 0<M<\infty, \epsilon>0 .
$$

This theorem may be deduced from Theorem 2. For any $z$ inside the domain bounded by the level curve $C_{e}$ we have 


$$
\frac{1}{2 \pi i} \int_{c_{\epsilon}} p(\zeta) \frac{1-\overline{\psi(z)} \psi(\zeta)}{\psi(\zeta)-\psi(z)} d \zeta=p(z) \frac{1-|\psi(z)|^{2}}{d \psi / d z}
$$

which for $\epsilon \rightarrow 0$ gives with (3.15)

$$
|p(z)|\left(1-|\psi(z)|^{2}\right) \leqq(M / 2 \pi)|d \psi / d z|, \quad z \in D .
$$

Denoting now

$$
\operatorname{Max}_{z \in c_{\epsilon}}|p(z)|=M(\epsilon),
$$

and using the fact that on $C_{\epsilon}|\psi(z)|=e^{-\epsilon}$ and that $d \psi / d z$ is bounded in $D$, it follows that

$$
\lim _{\epsilon \rightarrow 0} \epsilon^{2} M(\epsilon)=0 .
$$

But this is obviously the assumption of the nonoscillation analogue of Theorem 2 for simply-connected domains.

4. Non-Euclidean distance. Let us denote the non-Euclidean distance of any two points $z_{1}$ and $z_{2}$ in the unit circle $E$ by $\left|\left[z_{1} z_{2}\right]\right|$. This distance is defined by

$$
\left|\left[\begin{array}{ll}
z_{1} & z_{2}
\end{array}\right]\right|=\int_{\left[z_{1} z_{2}\right]} \frac{|d z|}{1-|z|^{2}},
$$

where the integration is along the orthogonal arc between $z_{1}$ and $z_{2}$ which we denote, as before, by $\left[z_{1} z_{2}\right]$.

We saw in Theorem 1 that condition (2.2) is not sufficient for nonoscillation. However, we obtain now-again by a modification of the proof leading to the sufficiency condition of Theorem I of [6]-the following theorem:

TheOREM 3. Let $p(z)$ be regular in $|z|<1$ and assume that

$$
|p(z)| \leqq a /\left(1-|z|^{2}\right)^{2}, \quad a>1, \text { for }|z|<1 .
$$

Let $y(z)$ be any (nontrivial) solution of

$$
y^{\prime \prime}(z)+p(z) y(z)=0,
$$

and assume that $y\left(z_{1}\right)=y\left(z_{2}\right)=0, z_{1} \neq z_{2},\left|z_{1}\right|<1,\left|z_{2}\right|<1$. Then

$$
\left|\left[\begin{array}{ll}
z_{1} & z_{2}
\end{array}\right]\right|>\log \frac{a^{1 / 2}+1}{a^{1 / 2}-1} .
$$

Moreover, the bound on the right-hand side of (4.3) is, for $a \rightarrow \infty$, of the correct order as a function of $a$.

Before proving this theorem, we remark that for $a \rightarrow 1$ the right-hand side of (4.3) $\rightarrow \infty$. But, indeed, $a=1$ corresponds to the sufficiency condition (1.6) 
of Theorem I of [6], which ensured that no solution $y(z)$ has more than one zero in $E$. Moreover, condition (2.1) of Theorem 1 implies (4.2) with $a=1 /\left(1-x_{0}^{2}\right)^{2}, 0<x_{0}<1$, so that whenever we apply this nonoscillation theorem we actually get also a lower bound for the non-Euclidean distance.

Proof. We choose again the transformation (2.3) so that $z_{1}$ and $z_{2}$ go into $\zeta= \pm \rho, 0<\rho<1$. By the invariance of the non-Euclidean distance, we have

$$
\left|\left[\begin{array}{ll}
z_{1} & z_{2}
\end{array}\right]\right|=\left|\left[\begin{array}{ll}
-\rho & \rho
\end{array}\right]\right|=\int_{-\rho}^{\rho} \frac{d x}{1-x^{2}}=\log \frac{1+\rho}{1-\rho} \quad(\zeta=x+i y) .
$$

(4.3) will therefore be established if we can show that

$$
\log \frac{1+\rho}{1-\rho}>\log \frac{a^{1 / 2}+1}{a^{1 / 2}-1}=\log \frac{1+1 / a^{1 / 2}}{1-1 / a^{1 / 2}},
$$

i.e., that

$$
\rho>1 / a^{1 / 2} .
$$

Assume, conversely, that

$$
0<\rho \leqq 1 / a^{1 / 2}
$$

This implies

$$
a \rho^{4} \leqq \rho^{2} .
$$

Moreover, it follows from $0<\rho<1$ that

$$
\left(\rho^{2}-x^{2}\right)^{2} / \rho^{4}<\left(1-x^{2}\right)^{2} \text { for }-\rho \leqq x \leqq \rho, x \neq 0 .
$$

Multiplying the last two inequalities we obtain

$$
a\left(\rho^{2}-x^{2}\right)^{2} \leqq \rho^{2}\left(1-x^{2}\right)^{2}, \quad-\rho \leqq x \leqq \rho
$$

with equality possible only at $x=0$.

By the transformation (2.3), equation (1.1) again transforms into

$$
y_{1}^{\prime \prime}(\zeta)+p_{1}(\zeta) y_{1}(\zeta)=0
$$

with a solution $y_{1}(\zeta) \not \equiv 0, y_{1}(\zeta)=u(\zeta)+i v(\zeta)$ such that $y_{1}( \pm \rho)=0$. As condition (4.2) is again invariant with respect to the transformation (2.3), it follows that

$$
\left|\int_{-\rho}^{\rho} p_{1}\left(u^{2}+v^{2}\right) d x\right| \leqq a \int_{-\rho}^{\rho} \frac{u^{2}+v^{2}}{\left(1-x^{2}\right)^{2}} d x \quad(\zeta=x+i y) .
$$

However, (4.6) implies

$$
a \int_{-\rho}^{\rho} \frac{u^{2}+v^{2}}{\left(1-x^{2}\right)^{2}} d x<\rho^{2} \int_{-\rho}^{\rho} \frac{u^{2}+v^{2}}{\left(\rho^{2}-: x^{2}\right)^{2}} d x
$$


so that

$$
\left|\int_{-\rho}^{\rho} p_{1}\left(u^{2}+v^{2}\right) d x\right|<\rho^{2} \int_{-\rho}^{\rho} \frac{u^{2}+v^{2}}{\left(\rho^{2}-x^{2}\right)^{2}} d x .
$$

But this last inequality is (2.10) and assumption (4.5) leads therefore to the contradiction which followed in $\$ 2$ from $(2.10)$. We have therefore proved the main assertion of the theorem.

To prove the second part of the theorem, we again use Hille's equation

$$
y^{\prime \prime}(z)+\frac{a}{\left(1-z^{2}\right)^{2}} y(z)=0,
$$

with $a=1+4 \gamma^{2}, \gamma>0$. Its solutions were given (see (2.11)) by

$$
y(z)=\left(z^{2}-1\right)^{1 / 2} \sin \left\{\gamma \log \left(\frac{1+z}{1-z}\right)-C\right\},
$$

which, as stated, have infinitely many zeros in $E$ if

$$
|\operatorname{Im}\{C\}|<\pi \gamma / 2 \text {. }
$$

These zeros are the points $z_{n}$ for which

$$
\gamma \log \left(\frac{1+z_{n}}{1-z_{n}}\right)-C=n \pi, \quad n=0, \pm 1, \pm 2, \cdots .
$$

By the transformation

$$
w=(1+z) /(1-z)
$$

$|z|<1$ is transformed into $\operatorname{Re}\{w\}>0$. To the zeros $z_{n}$ correspond in this half-plane the points $w_{n}$ given by

$$
w_{n}=e^{C / \gamma} \cdot e^{n \pi / \gamma}, \quad n=0, \pm 1, \cdots,
$$

which for $C$ satisfying (4.8) lie on a ray going through $w=0$ inside the halfplane. (4.10) implies now that for any such fixed solution (4.7), the nonEuclidean distance in the half-plane $\left|\left[w_{n} w_{n+1}\right]\right|$ is independent of the subscript $n$ and that for different solutions (4.7), $\left|\left[w_{n} w_{n+1}\right]\right|=\left|\left[z_{n} z_{n+1}\right]\right|$ is only a function of $|\operatorname{Im}\{C\}|$. It is easily checked that this function attains its minimum for $\operatorname{Im}\{C\}=0$ and we consider therefore the solutions (4.7) for which $C$ is real.

By (4.1) and (4.9) the non-Euclidean distance in $\operatorname{Re}\{w\}>0$ is given by

$$
\left|\left[\begin{array}{ll}
w^{\prime} w^{\prime \prime}
\end{array}\right]\right|=\frac{1}{2} \int_{\left[w^{\prime} w^{\prime \prime}\right]} \frac{|d w|}{u},
$$

where $w=u+i v$ and the integration is now along the arc orthogonal to $u=0$. 
It follows from (4.10) that for the zeros $z_{n}$ of the solutions (4.7) with real $C$

$$
\left|\left[\begin{array}{ll}
z_{n} & z_{n+1}
\end{array}\right]\right|=\left|\left[\begin{array}{ll}
w_{n} & w_{n+1}
\end{array}\right]\right|=\frac{1}{2} \int_{w_{n}}^{w_{n+1}} \frac{d u}{u}=\frac{\pi}{2 \gamma} .
$$

Substituting $1+4 \gamma^{2}$ for $a$ in the right-hand side of (4.3) we obtain, for $a \rightarrow \infty$,

$$
\log \frac{a^{1 / 2}+1}{a^{1 / 2}-1}=\log \frac{1+1 / a^{1 / 2}}{1-1 / a^{1 / 2}} \sim \frac{2}{a^{1 / 2}}=\frac{2}{\left(1+4 \gamma^{2}\right)^{1 / 2}} \sim \frac{1}{\gamma} .
$$

The ratio of the actual distance (4.11) to the bound given by our theorem converges therefore for $a \rightarrow \infty$ to $\pi / 2$. This concludes the proof of Theorem 3 .

Theorem 3 is a generalisation of the sufficiency part of Theorem I of [6]. The next theorem generalises now the necessity part of this theorem.

Theorem 4. Let $p(z)$ be regular in $|z|<1$ and assume that there exists a constant $a, a>1$, such that for any two zeros $z_{1}, z_{2}\left(z_{1} \neq z_{2},\left|z_{1}\right|<1,\left|z_{2}\right|<1\right)$ of any (nontrivial) solution $y(z)$ of

$$
y^{\prime \prime}(z)+p(z) y(z)=0
$$

the relation

$$
\left|\left[z_{1} z_{2}\right]\right| \geqq \log \frac{a^{1 / 2}+1}{a^{1 / 2}-1}
$$

holds. Then

$$
|p(z)|<3 a /\left(1-|z|^{2}\right)^{2} \quad \text { for }|z|<1 .
$$

Moreover, the bound on the right-hand side of (4.13) is, for $a \rightarrow \infty$, of the correct order as a function of $a$.

Proof. Let us denote

$$
\log \frac{a^{1 / 2}+1}{a^{1 / 2}-1}=\log \frac{1+1 / a^{1 / 2}}{1-1 / a^{1 / 2}}=2 r .
$$

Condition (4.12) is equivalent to the statement that every function $f(z)$, defined as the ratio of two independent solutions of (1.1), is univalent in every non-Euclidean circle of radius $r$. Choosing now this circle so that its center is at $z=0$, it follows from (4.14) and (4.4) that its Euclidean radius is given by $1 / a^{1 / 2}$. Let now

$$
\begin{gathered}
z=z^{\prime}\left(1 / a^{1 / 2}\right) . \\
f(z)=f\left(z^{\prime}\left(1 / a^{1 / 2}\right)\right)=g\left(z^{\prime}\right), \quad|z|<1 / a^{1 / 2} .
\end{gathered}
$$

It follows that

$$
\{f(z), z\}=a\left\{g\left(z^{\prime}\right), z^{\prime}\right\} .
$$


$g\left(z^{\prime}\right)$ is univalent in $\left|z^{\prime}\right|<1$ and it follows from (1.5) that

$$
\left|\left\{g\left(z^{\prime}\right), z^{\prime}\right\}\right|_{z^{\prime}-0} \leqq 6 .
$$

However, the equality sign will hold here only if $g\left(z^{\prime}\right)$ is the Koebe extremal function or one of its linear transforms. But these functions have either a double pole or a zero of their derivative on $\left|z^{\prime}\right|=1$. The corresponding $f(z)$ could therefore not be of the restricted class in $|z|<1$. It follows that

$$
|\{f(z), z\}|_{z=0}<6 a \text {. }
$$

Let now $E_{1}$ be a non-Euclidean circle of center $z=z_{0}\left(\left|z_{0}\right|<1\right)$ and radius $r$. The transformation

$$
z=\left(\zeta+z_{0}\right) /\left(1+\bar{z}_{0} \zeta\right)
$$

maps $E_{1}$ onto $|\zeta|<1 / a^{1 / 2}$. Since $f(z)$ was univalent in $E_{1}$, it follows that the same is true of $g(\zeta)=f\left(\left(\zeta+z_{0}\right) /\left(1+\bar{z}_{0} \zeta\right)\right)$ in $|\zeta|<1 / a^{1 / 2}$. (4.15) implies therefore

$$
|\{g(\zeta) . \zeta\}|_{\zeta=0}<6 a,
$$

and it follows from (2.9) that

$$
|\{f(z), z\}|_{z=z_{0}}\left(1-\left|z_{0}\right|^{2}\right)^{2}<6 a .
$$

But this clearly entails (4.13) and we have proved the main assertion of the theorem.

The proof of the second part of the theorem uses again Hille's example, this time for $a<-3\left(^{5}\right)$. Setting, therefore, $a=1-4 \gamma^{2}, \gamma>1$, the solutions of (2.12) will be given by

$$
y(z)=\left(z^{2}-1\right)^{1 / 2} \sin \{i \gamma \log ((1+z) /(1-z))-C\} .
$$

We first map $|z|<1$ onto $\operatorname{Re}\{w\}>0$ by means of (4:9). This half -plane is then mapped by

$$
\zeta=i \gamma \cdot \log w, \quad \zeta=x+i y,
$$

onto the strip $|x|<\pi \gamma / 2$, and it follows that, for $\gamma>1$, some solutions (4.16) will have at least two zeros in $E$. (For $\gamma>2$, every solution will have at least two zeros and so on.) Two "successive" zeros $z_{1}$ and $z_{2}$ of such a solution correspond to points $\zeta_{1}$ and $\zeta_{2}$ in the strip, such that

$$
\zeta_{2}-\zeta_{1}=\pi
$$

The corresponding points in the half-plane will, therefore, be connected by

$$
w_{1}=w_{2} e^{i \pi / \gamma} \text {. }
$$

(b) Note that now Hille's parameter $a$ is not the constant appearing in the statement of our theorem (but is equal to -3 times this constant). 
For any two such points $w_{1}$ and $w_{2},\left|\left[w_{1} w_{2}\right]\right|$ will be only a function of their argument and this function attains its minimum in the symmetric case. Setting $w_{1}^{*}=e^{i \pi / 2 \gamma}, w_{2}^{*}=e^{-i \pi / 2 \gamma}$ we obtain

$$
\begin{aligned}
\left|\left[\begin{array}{ll}
z_{1} & z_{2}
\end{array}\right]\right|=\left|\left[\begin{array}{ll}
w_{1} & w_{2}
\end{array}\right]\right| \geqq\left|\left[\begin{array}{ll}
w_{1}^{*} & w_{2}^{*}
\end{array}\right]\right| & =\frac{1}{2} \int_{\left[w_{1}^{*} w_{2}^{*}\right]} \frac{|d w|}{u}=\frac{1}{2} \int_{-\pi / 2 \gamma}^{\pi / 2 \gamma} \frac{d \theta}{\cos \theta} \\
& =\frac{1}{2} \log \frac{1+\sin (\pi / 2 \gamma)}{1-\sin (\pi / 2 \gamma)},
\end{aligned}
$$

and this lower bound for $\left|\left[z_{1} z_{2}\right]\right|$ is actually attained by all functions (4.16) for which $\operatorname{Re}\{C\}=-\pi / 2$.

For $\gamma \rightarrow \infty$,

$$
\frac{1}{2} \log \frac{1+\sin (\pi / 2 \gamma)}{1-\sin (\pi / 2 \gamma)} \sim \log \frac{4 \gamma / \pi+1}{4 \gamma / \pi-1},
$$

so that by our theorem the coefficient of $\left(1-|z|^{2}\right)^{-2}$ in the bound for $p(z)$ would be asymptotically equal to $48 \gamma^{2} / \pi^{2}$. However, the actual coefficient is $\left(4 \gamma^{2}-1\right)$, so that their ratio converges for $\gamma \rightarrow \infty$ to $12 / \pi^{2}$. This concludes the proof of Theorem 4.

We see that if in Hille's example (2.12) the parameter $a$ moves to the left from -3 along the negative real axis, we successively obtain solutions $y(z)$ with more and more zeros in $E$; however, the equation will always remain nonoscillatory. It follows therefore that no condition of the form

$$
|p(z)|<C /\left(1-|z|^{2}\right)^{2},
$$

with given $C$, is necessary for nonoscillation of (1.1).

The two theorems of this section may be considered as conditions for functions $f(z)$, meromorphic in $E$, to be there "locally univalent of nonEuclidean modulus $r$. " Such functions are by their definition in the restricted class. The corresponding Euclidean class of functions was first considered by Montel [5].

5. Euclidean distance I. We consider again functions $p(z)$ regular in $E$ and the zeros of any solution $y(z)$ of equation (1.1). We saw that the existence of a common positive lower bound for the non-Euclidean distance of two zeros is equivalent to the assumption

$$
p(z)=O\left(1 /\left(1-|z|^{2}\right)^{2}\right) .
$$

Clearly, conditions on $p(z)$ which ensure similarly the existence of a common lower bound for the Euclidean distance must be more restringent. Indeed, Hille's example (2.12) shows that the existence of a non-Euclidean bound does not imply the existence of an analogous Euclidean bound, even in the case (corresponding to $-15 \leqq a<-3$ ) where every solution $y(z)$ has at most two zeros in $E$. 
THEOREM 5. Let $p(z)$ be regular in $|z|<1$, and set

$$
M(t)=\underset{|z|-\imath}{\operatorname{Max}}|p(z)|, \quad 0 \leqq t<1 .
$$

Assume that

$$
M(t) \leqq 1 /\left(1-t^{2}\right) \quad \text { for } r \leqq t<1,0<r<1 .
$$

Let $y(z)$ be any (nontrivial) solution of

$$
y^{\prime \prime}(z)+p(z) y(z)=0
$$

and assume that $y\left(z_{1}\right)=y\left(z_{2}\right)=0, z_{1} \neq z_{2},\left|z_{1}\right|<1,\left|z_{2}\right|<1$. Then

$$
\left|z_{1}-z_{2}\right| \geqq d
$$

where

$$
d=2\left(1-r^{2}\right)^{1 / 2} .
$$

Proof. We assume that (5.2) holds and that there exists a solution $y(z)$ of $(1.1)(y(z) \not \equiv 0)$ such that

$$
\left|z_{1}-z_{2}\right|=\delta<d .
$$

Multiplying (1.1) by $\bar{y} d z$ and integrating by parts from $z_{1}$ to $z_{2}$ along a path in $E$ we obtain

$$
\left[\bar{y} y^{\prime}\right]_{z_{1}}^{s_{2}}-\int_{y_{1}}^{z_{2}}\left|y^{\prime}\right|^{2} \overline{d z}+\int_{z_{1}}^{z_{2}} p|y|^{2} d z=0,
$$

the "Green's transform" of (1.1). Using now $y\left(z_{1}\right)=y\left(z_{2}\right)=0$ and choosing as path the segment $\left\langle z_{1}, z_{2}\right\rangle$ (whose length element we denote by $d \sigma$ ) we obtain

$$
\int_{z_{1}}^{z_{2}}\left|y^{\prime}\right|^{2} d \sigma \leqq \int_{z_{1}}^{z_{2}}|p||y|^{2} d \sigma .
$$

We shall reach the desired contradiction by three consecutive transformations of this inequality; these transformations result from very simple transformations of the segment $\left\langle z_{1}, z_{2}\right\rangle$.

For the first transformation choose $a, 0<a<1$, such that $\left|z_{1}\right|<a,\left|z_{2}\right|<a$ and such that, setting

$$
\left.\rho=\left(a^{2}-\delta^{2} / 4\right)^{1 / 2} \quad \text { (i.e., } \delta / 2=\left(a^{2}-\rho^{2}\right)^{1 / 2}\right),
$$

we have

$$
r<\rho .
$$

This is clearly possible by (5.4) and (5.5). We move the segment $\left\langle z_{1}, z_{2}\right\rangle$ parallel to itself in the direction given by the normal from $z=0$ to the line 
containing $\left\langle z_{1}, z_{2}\right\rangle$ until one endpoint lies on $|z|=a$. Then (in case the second endpoint is still inside $|z|=a$ ) we rotate the segment about the first endpoint so as to bring also the second end point onto $|z|=a$ (and we choose the smaller of the two possible rotations accomplishing this). In other words, we moved the segment $\left\langle z_{1}, z_{2}\right\rangle$ in such a way that it became a chord in $|z|=a$ and it is obvious that during this motion the distance of each point from $z=0$ increased. The distance of this chord from the origin is $\rho$. If we denote the length coordinate of the chord, measured from its centre, by $s\left(-\left(a^{2}-\rho^{2}\right)^{1 / 2}\right.$ $\left.\leqq s \leqq\left(a^{2}-\rho^{2}\right)^{1 / 2}\right)$, then the distance of the point with the coordinate $s$ from $z=0$ will be $\left(\rho^{2}+s^{2}\right)^{1 / 2}$.

We define now $y_{1}(s)$ on the chord by giving that function the same values which $y(z)$ took at the corresponding points of the segment $\left\langle z_{1}, z_{2}\right\rangle$; similarly we define $p_{1}(s)$ by the values of $p(z)$ on $\left\langle z_{1}, z_{2}\right\rangle . y_{1}(s)$ is therefore analytic for $-\left(a^{2}-\rho^{2}\right)^{1 / 2} \leqq s \leqq\left(a^{2}-\rho^{2}\right)^{1 / 2}$ and $y_{1}\left( \pm\left(a^{2}-\rho^{2}\right)^{1 / 2}\right)=0$. As $M(t)$ is, by the maximum principle, a nondecreasing function of $t$, it follows from the above remark about the increasing distances from the origin that

$$
\left|p_{1}(s)\right| \leqq M\left(\left(\rho^{2}+s^{2}\right)^{1 / 2}\right), \quad-\left(a^{2}-\rho^{2}\right)^{1 / 2} \leqq s \leqq\left(a^{2}-\rho^{2}\right)^{1 / 2} .
$$

(5.6) implies therefore

$$
\int_{-\left(a^{2}-\rho^{2}\right)^{1 / 2}}^{\left(a^{2}-\rho^{2}\right)^{1 / 2}}\left|\frac{d y_{1}}{d s}\right|^{2} d s \leqq \int_{-\left(a^{2}-\rho^{2}\right)^{1 / 2}}^{\left(a^{2}-\rho^{2}\right)^{1 / 2}} M\left(\left(\rho^{2}+s^{2}\right)^{1 / 2}\right)\left|y_{1}(s)\right|^{2} d s .
$$

Our second transformation maps now half the chord, i.e., $0 \leqq s \leqq\left(a^{2}-\rho^{2}\right)^{1 / 2}$, linearly on to the segment $\rho \leqq t \leqq a$ of the real axis so that $s=0$ is carried into $t=\rho$ and $s=\left(a^{2}-\rho^{2}\right)^{1 / 2}$ into $t=a$. Similarly, we map $-\left(a^{2}-\rho^{2}\right)^{1 / 2} \leqq s \leqq 0$ onto $-a \leqq t \leqq-\rho$. These transformations are given by

$$
t= \pm \rho+\frac{a-\rho}{\left(a^{2}-\rho^{2}\right)^{1 / 2}} s \quad \text { for } 0 \leqq \pm s \leqq\left(a^{2}-\rho^{2}\right)^{1 / 2} .
$$

It is easily seen that

$$
\left(\rho^{2}+s^{2}\right)^{1 / 2} \leqq \rho \pm \frac{a-\rho}{\left(a^{2}-\rho^{2}\right)^{1 / 2}} s \quad \text { for } 0 \leqq \pm s \leqq\left(a^{2}-\rho^{2}\right)^{1 / 2}, 0<\rho<a,
$$

where the sign of equality holds only for $s=0, \pm\left(a^{2}-\rho^{2}\right)^{1 / 2}$. By $(5.10)$ this shows that under this second transformation the distance of each point from the origin again increases, except for the points $s=0, \pm\left(a^{2}-\rho^{2}\right)^{1 / 2}$ whose distance remains constant.

The function $Y(t)$ defined by

$$
Y(t)=Y\left( \pm \rho+\frac{a-\rho}{\left(a^{2}-\rho^{2}\right)^{1 / 2}} s\right)=y_{1}(s)
$$

will thus have the following properties: $Y(t)$ is analytic on the segments 
$-a \leqq t \leqq-\rho$ and $\rho \leqq t \leqq a ; Y(a)=Y(-a)=0 ; Y(t)$ and all its derivatives take the same values at $t=\rho$ and $t=-\rho$. Defining

$$
M(t)=M(-t) \quad \text { for }-1<t<0,
$$

and observing that the distances from the origin do not decrease under this second transformation, we obtain from (5.9)

$$
\begin{aligned}
\int_{-a}^{-\rho}\left|\frac{d Y}{d t}\right|^{2} d t+\int_{\rho}^{a}\left|\frac{d Y}{d t}\right|^{2} d t \leqq & \frac{a+\rho}{a-\rho}\left\{\int_{-a}^{-\rho} M(t)|Y(t)|^{2} d t\right. \\
& \left.+\int_{\rho}^{a} M(t)|Y(t)|^{2} d t\right\} .
\end{aligned}
$$

We use now the inequality

$$
\frac{1}{1-t^{2}}<\frac{a-\rho}{a+\rho} \frac{2}{(a-\rho)^{2}-(t-\rho)^{2}}, \quad 0<\rho \leqq t \leqq a<1, \rho<a,
$$

which will be established at the end of the proof. By our assumption (5.2), and in view of (5.8) and (5.11), it follows now that

$$
M(t) \leqq \frac{1}{1-t^{2}} \quad \text { for }-a \leqq t \leqq-\rho \text { and } \rho \leqq t \leqq a .
$$

The last three inequalities (and (5.11)) yield

$$
\int_{-a}^{-\rho}\left|\frac{d Y}{d t}\right|^{2} d t+\int_{\rho}^{a}\left|\frac{d Y}{d t}\right|^{2} d t<\int_{-a}^{-\rho} g(t)|Y(t)|^{2} d t+\int_{\rho}^{a} g(t)|Y(t)|^{2} d t
$$

where

$$
g(t)=\frac{2}{(a-\rho)^{2}-(t \mp \rho)^{2}} \quad \text { for } \rho \leqq \pm t \leqq a .
$$

By assumption $y(z)$ was a nontrivial solution of $(1.1)$ and therefore $Y(t) \not \equiv 0$. Moreover, we may exclude the trivial case $M(t) \equiv 0$ (i.e., $p(z) \equiv 0)$ and we are therefore by (5.13) justified in excluding the equality sign in (5.14).

The third transformation translates the two segments $\langle-a,-\rho\rangle$ and $\langle\rho, a\rangle$ of the real axis until they meet at the origin, i.e., we introduce the variable $x$ by

$$
x=t \mp \rho \quad \text { for } \rho \leqq \pm t \leqq a .
$$

With the notation

$$
a-\rho=b,
$$

it follows that $x$ varies between $-b$ and $b$. Defining now $g_{1}(x)=g_{1}(t \mp \rho)=g(t)$, we have 


$$
g_{1}(x)=\frac{2}{b^{2}-x^{2}},
$$

Similarly, we define $Y_{1}(x)=Y_{1}(t \mp \rho)=Y(t)$ and it follows that $Y_{1}(x)$ is analytic for $-b \leqq x \leqq b$ and $Y_{1}( \pm b)=0$. (5.14) transforms therefore into

$$
\int_{-b}^{b}\left|\frac{d Y_{1}}{d x}\right|^{2} d x<2 \int_{-b}^{b} \frac{\left|Y_{1}(x)\right|^{2}}{b^{2}-x^{2}} d x .
$$

We now use the integral inequality

$$
2 \int_{-b}^{b} \frac{u^{2} d x}{b^{2}-x^{2}} \leqq \int_{-b}^{b} u^{\prime 2} d x, \quad u=u(x),
$$

which holds for continuously differentiable real functions $u(x)$ having at $x= \pm b$ zeros of the first order $\left.{ }^{6}\right)$. (5.16) follows from the semi-definiteness of the integral

$$
\int_{-b}^{b}\left(u^{\prime}+\frac{2 x u}{b^{2}-x^{2}}\right)^{2} d x
$$

Expanding and integrating by parts, we obtain

$$
\int_{-b}^{b} u^{\prime 2} d x+\left.2 \frac{x u^{2}}{b^{2}-x^{2}}\right|_{-b} ^{b}-2 \int_{-b}^{b} \frac{\left(b^{2}+x^{2}\right) u^{2}}{\left(b^{2}-x^{2}\right)^{2}} d x+4 \int_{-b}^{b} \frac{x^{2} u^{2}}{\left(b^{2}-x^{2}\right)^{2}} d x \geqq 0 .
$$

$u$ being $O(b-x)$ and $O(b+x)$ and $x=b$ and $x=-b$ respectively, the integrals exist and the integrated part vanishes, which proves (5.16). Writing now $Y_{1}(x)=u(x)+i v(x)$ and applying (5.16) to both $u(x)$ and $v(x)$, we obtain the desired contradiction to (5.15).

It remains to prove the inequality

$$
\frac{1}{1-t^{2}}<\frac{a-\rho}{a+\rho} \frac{2}{(a-\rho)^{2}-(t-\rho)^{2}}, \quad 0<\rho \leqq t \leqq a<1, \rho<a .
$$

For $a=t$ the right-hand side becomes infinite so that we may assume $0<\rho$ $\leqq t<a<1$. Since

$$
\frac{1}{1-t^{2}}<\frac{1}{a^{2}-t^{2}}
$$

it will suffice to show that

$$
\frac{1}{a^{2}-t^{2}} \leqq \frac{2(a-\rho)}{(a+\rho)\left[(a-\rho)^{2}-(t-\rho)^{2}\right]}=\frac{2(a-\rho)}{(a+\rho)(a+t-2 \rho)(a-t)}
$$

i.e., that

( ${ }^{\circ}$ See Hardy, Littlewood, P6lya [2, p. 193]. 


$$
\frac{1}{a+t} \leqq \frac{2(a-\rho)}{(a+\rho)(a+t-2 \rho)} .
$$

This inequality is equivalent to $(a+\rho)(a+t-2 \rho) \leqq 2(a-\rho)(a+t)$, which by computing the products and rearrangement of terms reduces to

$$
(a-\rho)^{2}+\rho^{2}+a \rho+a t-3 \rho t \geqq 0 .
$$

To prove the last inequality it will be enough to show that, for fixed $\rho$ and $a$ $(0<\rho<a<1)$ and for all $t, \rho \leqq t \leqq a$, the function

$$
f(t)=\rho^{2}+a \rho+a t-3 \rho t
$$

is positive. However, $f(t)$ is positive at the endpoints of the interval $\langle\rho, a\rangle$ and, being a linear function of $t$, also positive inside the interval. Thus (5.17) is proved and the inequality (5.13) is established. This completes the proof of Theorem 5.

We remark that without any modification our proof holds also in the case $r=0$. Assumption (5.2) becomes then

$$
M(t) \leqq 1 /\left(1-t^{2}\right) \quad \text { for } 0 \leqq t<1,
$$

and the conclusion is now that no solution $y(z)$ of (1.1) has more than one zero in $|z|<1$. But this is clearly a consequence of the sufficiency part of Theorem I of [6] and also of a criterion announced by Pokornyi [10], stating that

$$
M(t) \leqq 2 /\left(1-t^{2}\right) \quad \text { for } 0 \leqq t<1,
$$

is sufficient to ensure the same conclusion. This criterion is sharp, i.e., the constant 2 cannot be increased.

However, we show now that, similar to the theorems on the non-Euclidean distance, Theorem 5 is of the "correct order" for $r \rightarrow 1$. In view of the geometrical meaning of $d$ and $r$ (length of chord and its distance from the origin) it seems natural not to change definition (5.4). We have then the following statement:

No condition of the form

$$
M(t) \leqq C /\left(1-t^{2}\right)^{\lambda}, \quad \lambda>1, C>0, r \leqq t<1,
$$

is, for all $r(0 \leqq r<1)$, sufficient to ensure that

$$
\left|z_{1}-z_{2}\right| \geqq d \text {. }
$$

Here

$$
d=2\left(1-r^{2}\right)^{1 / 2},
$$

and $z_{1}, z_{2}$ are again any pair of zeros of an arbitrary solution of (1.1).

To prove this consider 


$$
p(z) \equiv C_{1}
$$

The distance $d^{\prime}$ between neighboring zeros of any solution of (1.1) is then

$$
d^{\prime}=\pi / C_{1}^{1 / 2} \text {. }
$$

Condition (5.19) holds now for $r \leqq t<1$, where $r$ is given by

$$
\left(1-r^{2}\right)^{\lambda}=C / C_{1} \text {. }
$$

The bound $d$, given by (5.4), becomes

$$
d=2\left(1-r^{2}\right)^{1 / 2}=2\left(C / C_{1}\right)^{1 / 2 \lambda} .
$$

As $\lambda>1$, the lower bound $d$ would, for large $C_{1}$, be larger than the actual distance $d^{\prime}$ and we have proved the above statement.

We mentioned that for $r=0$, condition (5.18) is sharp. It follows that in Theorem 5 condition (5.2) cannot be replaced by a condition of the form

$$
M(t) \leqq C /\left(1-t^{2}\right), \quad r \leqq t<1,0 \leqq r<1,
$$

with $C>2$. Recently P. R. Beesack (in a forthcoming Washington University thesis) has shown that the conclusion indeed holds with $C=2$.

\section{Euclidean distance II.}

Theorem 6. Let $p(z)$ be regular and bounded for $|z|<1$, and set $M(x)$ $=\operatorname{Max}_{|z|=x}|p(z)|(0 \leqq x<1) ;$ let $M(1)=\lim _{x \rightarrow 1} M(x)$. For given $d, 0<d \leqq 2$, let

$$
r=\left(1-d^{2} / 4\right)^{1 / 2}
$$

and define $M_{d}(x)$ for $-d / 2<x<d / 2$ as follows:

In Case I: $0<d \leqq 2^{1 / 2}$,

$$
\begin{array}{lr}
M_{d}(x)=M(1) & \text { for } 1-r \leqq x<d / 2, \\
M_{d}(x)=M(x+r) & \text { for } 0 \leqq x<1-r, \\
M_{d}(x)=M_{d}(-x) & \text { for }-d / 2<x<0 .
\end{array}
$$

In Case II : $2^{1 / 2}<d \leqq 2$,

$$
\begin{array}{rlr}
M_{d}(x) & =M(1) & \text { for } 1-\left(2^{1 / 2}-d / 2\right) \leqq x<d / 2, \\
\left(6.2^{\prime \prime}\right) & M_{d}(x)=M\left(x+2^{1 / 2}-d / 2\right) & \text { for } 0 \leqq x<1-\left(2^{1 / 2}-d / 2\right), \\
M_{d}(x) & =M_{d}(-x) & \text { for }-d / 2<x<0 .
\end{array}
$$

Assume that the differential equation

$$
y^{\prime \prime}(x)+M_{d}(x) y(x)=0
$$

has a (real) solution $y(x)$ such that $y(x) \neq 0$ for $-d / 2<x<d / 2$. Then, in case $d=2$, no (nontrivial) solution $y(z)$ of (1.1) has two zeros in $|z|<1$, while in case 
$0<d<2,(5.3)$ holds. $z_{1}, z_{2}$ are again any pair of zeros in $|z|<1$ of an arbitrary nontrivial solution $y(z)$ of (1.1).

Proof. (6.1) is equivalent to (5.4); the connection between $d$ and $r$ has the same geometrical meaning as in Theorem 5 . We remark that for $0<d \leqq 2$

$$
\frac{d}{2}+r=\frac{d}{2}+\left(1-d^{2} / 4\right)^{1 / 2} \leqq 2^{1 / 2},
$$

with equality only for $d=r=2^{1 / 2} / 2$. Therefore

$$
r \leqq 2^{1 / 2}-d / 2 \text {, }
$$

and it follows that $M_{d}(x)$ in Case II, defined by $\left(6.2^{\prime \prime}\right)$, is not smaller than if it were also defined in this case by $\left(6.2^{\prime}\right)$.

We suppose that for a fixed value of the parameter $d$ the assumption of the theorem holds and that there exists a nontrivial solution $y(z)$ of (1.1) such that $y\left(z_{1}\right)=y\left(z_{2}\right)=0, z_{1} \neq z_{2},\left|z_{1}\right|<1,\left|z_{2}\right|<1$, and such that (5.5) holds. Using the Green's transform along the segment $\left\langle z_{1}, z_{2}\right\rangle$ we obtain (5.6). We shall reach the desired contradiction again by three consecutive transformations of (5.6).

The first transformation is identical to the first transformation used in the last proof. We use the same notation as before and go through the same steps. The only modification is that we choose now $a$ so near to 1 that, in addition to all the former requirements, we have now also

$$
\rho+\delta / 2>1 \text {, }
$$

where $\rho$ is again defined by (5.7). Except for a slight change in notation, our result was as follows: On the chord (in $|z|=a$, of length $\delta$ and of distance $\rho$ from the origin) there exists a nontrivial analytic function $y_{1}(s),-\delta / 2 \leqq s$ $\leqq \delta / 2$, such that $y_{1}( \pm \delta / 2)=0$ and such that

$$
\int_{-\delta / 2}^{\delta / 2}\left|\frac{d y_{1}}{d s}\right|^{2} d s \leqq \int_{-\delta / 2}^{\delta / 2} M\left(\left(\rho_{=}^{2}+s^{2}\right)^{1 / 2}\right)\left|y_{1}(s)\right|^{2} d s .
$$

The second transformation displaces now half the chord, i.e., $0 \leqq s \leqq \delta / 2$, so that it coincides with the segment $\rho \leqq t \leqq \rho+\delta / 2$ on the real axis. Similarly, we transform $-\delta / 2 \leqq s \leqq 0$ into $-(\rho+\delta / 2) \leqq t \leqq-\rho$. These transformations are given by $t= \pm \rho+s$, and it is obvious that the distance of each point from the origin increases, except for the point $s=0$ whose distance remains constant. Defining now $Y(t)=Y( \pm \rho+s)=y_{1}(s)$, we obtain a function $Y(t)$ analytic on the segments $-(\rho+\delta / 2) \leqq t \leqq-\rho$ and $\rho \leqq t \leqq \rho+\delta / 2$, such that $Y( \pm(\rho+\delta / 2))=0$ and $Y(\rho)=Y(-\rho)$. Setting $M(t)=M(1)$ for $1<t \leqq \rho+\delta / 2$ (see (6.4)) and extending $M(t)$ by symmetry to negative values of $t$, we obtain from (6.5) 


$$
\begin{aligned}
\int_{-(\rho+\delta / 2)}^{-\rho}\left|\frac{d Y}{d t}\right|^{2} d t+\int_{\rho}^{\rho+\delta / 2}\left|\frac{d Y}{d t}\right|^{2} d t< & \int_{-(\rho+\delta / 2)}^{-\rho} M(t)|Y(t)|^{2} d t \\
& +\int_{\rho}^{\rho+\delta / 2} M(t)|Y(t)|^{2} d t .
\end{aligned}
$$

The proper inequality sign is justified by the remark following (5.14).

The third transformation is similar to the third transformation used in the proof of Theorem 5. We translate the two segments symmetrically towards the origin; however, this time not until they meet. Moreover, we have to distinguish between the two cases according to the value of $d$.

CASE I. $0<d \leqq 2^{1 / 2}$.

In this case it follows (by (5.4), (5.5), and (5.7)) that

$$
\begin{aligned}
\delta / 2+\rho & =\delta / 2+\left(a^{2}-\delta^{2} / 4\right)^{1 / 2}<\delta / 2+\left(1-\delta^{2} / 4\right)^{1 / 2}<d / 2+\left(1-d^{2} / 4\right)^{1 / 2} \\
& =d / 2+r .
\end{aligned}
$$

The last inequality follows from the fact that the function $d / 2+\left(1-d^{2} / 4\right)^{1 / 2}$ is increasing for $0 \leqq d \leqq 2^{1 / 2}$. We have therefore

$$
\delta / 2+\rho<d / 2+r .
$$

In this case we displace both segments by $r=\left(1-d^{2} / 4\right)^{1 / 2}$, i.e., we introduce $x$ by $x=t \mp r$ for $\rho \leqq \pm t \leqq \rho+\delta / 2$ and define again $Y_{1}(x)=Y_{1}(t \mp r)=Y(t)$ and $M^{\prime}(x)=M^{\prime}(t \mp r)=M(t)$ for $\rho-r \leqq \pm x \leqq \rho+\delta / 2-r$. By (5.8), $\rho-r>0$, and by (6.7), $\rho+\delta / 2-r<d / 2$ and it follows by $\left(6.2^{\prime}\right)$ that in the two intervals just considered $M^{\prime}(x)=M_{d}(x)$.

We now continue the function $Y_{1}(x)$ across the gap $-(\rho-r)<x<\rho-r$ between the two intervals by setting $Y_{1}(x)=Y_{1}(\rho-r)$ for those $x$. With the notation

$$
\rho+\delta / 2-r=b^{\prime} \quad\left(0<b^{\prime}<d / 2\right),
$$

we see that $Y_{1}(x)$ is continuous in $-b^{\prime} \leqq x \leqq b^{\prime}$, analytic there except at the points $x= \pm(\rho-r)$, and $Y_{1}\left( \pm b^{\prime}\right)=0$. Using now that $d Y_{1} / d x \equiv 0$ for $-(\rho-r)$ $<x<\rho-r$ we obtain from $(6.6)$

$$
\int_{-b^{\prime}}^{b^{\prime}}\left|\frac{d Y_{1}}{d x}\right|^{2} d x<\int_{-b^{\prime}}^{b^{\prime}} M_{d}(x)\left|Y_{1}(x)\right|^{2} d x .
$$

CASE II: $2^{1 / 2}<d \leqq 2$.

Consider first the Case $\mathrm{II}_{a}$ given by

$$
\delta / 2+\rho<d / 2+r .
$$

In this case we proceed exactly as under Case I. We therefore arrive again at the inequality $\left(6.8^{\prime}\right)$ where, however, $M_{d}(x)$ is defined by $\left(6.2^{\prime}\right)$ instead of by $\left(6.2^{\prime \prime}\right.$ ) (as it should be in Case II). But using now the remark made at the 
beginning of this proof, it follows that, in Case $\mathrm{II}_{a},\left(6.8^{\prime}\right)$ holds with the proper definition of $M_{d}(x)$.

The case

$$
\delta / 2+\rho=d / 2+r
$$

may be excluded, by slightly changing $a$, and there remains therefore only the Case $\mathrm{II}_{b}$,

$$
\delta / 2+\rho>d / 2+r .
$$

In this case we choose $\epsilon>0$ such that

$$
\epsilon<d / 2-\delta / 2
$$

and

$$
\rho+\delta / 2+\epsilon<2^{1 / 2} .
$$

We translate now both segments by $\tau=\rho+\delta / 2-d / 2+\epsilon$, where by (6.9) and the last inequality

$$
0<\tau<2^{1 / 2}-d / 2 \text {. }
$$

In other words, we transform by $x=t \mp \tau$ the segments $\rho \leqq \pm t \leqq \rho+\delta / 2$ into the segments $d / 2-\delta / 2-\epsilon \leqq \pm x \leqq d / 2-\epsilon$ and define there $Y_{1}(x)=Y_{1}(t \mp \tau)$ $=Y(t), M^{\prime \prime}(x)=M^{\prime \prime}(t \mp \tau)=M(t)$. By $\left(6.2^{\prime \prime}\right)$ and (6.10) it follows that $M^{\prime \prime}(x) \leqq M_{d}(x)$. Defining again $Y_{1}(x)=Y_{1}(d / 2-\delta / 2-\epsilon)$ for $-(d / 2-\delta / 2-\epsilon)$ $<x<(d / 2-\delta / 2-\epsilon)$ and calling

$$
d / 2-\epsilon=b^{\prime \prime} \quad\left(0<b^{\prime \prime}<d / 2\right),
$$

it follows that $Y_{1}(x)$ is continuous and piecewise analytic in $-b^{\prime \prime} \leqq x \leqq b^{\prime \prime}$, $Y_{1}\left( \pm b^{\prime \prime}\right)=0$ and

$$
\int_{-b^{\prime \prime}}^{b^{\prime \prime}}\left|\frac{d Y_{1}}{d x}\right|^{2} d x<\int_{-b^{\prime \prime}}^{b^{\prime \prime}} M_{d}(x)\left|Y_{1}(x)\right|^{2} d x .
$$

We reached in each case the same conclusion. There exists a complex function $Y_{1}(x)$ which is continuous and piecewise analytic in $-b \leqq x \leqq b \quad(0<b$ $<d / 2)$, which vanishes at the endpoints of this interval and for which

$$
\int_{-b}^{b}\left|\frac{d Y_{1}}{d x}\right|^{2} d x<\int_{-b}^{b} M_{d}(x)\left|Y_{1}(x)\right|^{2} d x .
$$

(6.8) will lead to the desired contradiction to our assumption $\left({ }^{7}\right)$. Indeed, let $\lambda$ be the lowest eigenvalue of the differential system

$$
y^{\prime \prime}(x)+\lambda M_{d}(x) y(x)=0, \quad y(b)=y(-b)=0 .
$$

(') See Nehari [8]. 
It follows by the minimum property of this eigenvalue that for any real function $u(x)$, which is continuous and piecewise smooth in $-b \leqq x \leqq b$ and which vanishes at $x= \pm b$,

$$
\int_{-b}^{b} M_{d}(x) u(x)^{2} d x \leqq \frac{1}{\lambda} \int_{-b}^{b} u^{\prime 2} d x .
$$

Splitting now $Y_{1}(x)$ into its real and imaginary part, we obtain by (6.8) and (6.11) that

$$
\int_{-b}^{b}\left|\frac{d Y_{1}}{d x}\right|^{2} d x<\frac{1}{\lambda} \int_{-b}^{b}\left|\frac{d Y_{1}}{d x}\right|^{2} d x
$$

i.e., that $\lambda<1$. By the Sturm comparison theorem it follows that the solution $y(x)$ of equation (6.3) for which $y(-b)=0$ has another zero in $-b<x<b$. But as $-d / 2<-b<b<d / 2$ this contradicts, by the Sturm separation theorem, our assumption. Theorem 6 is therefore established.

Let now $M(1)=C>0$. As the solution $y=\cos C^{1 / 2} x$ of the differential equation $y^{\prime \prime}(x)+C y(x)=0$ is $\neq 0$ for $-\pi / 2 C^{1 / 2}<x<\pi / 2 C^{1 / 2}$, it follows from the Sturm comparison theorem that the assumption of Theorem 6 is satisfied for $d=\operatorname{Min}\left(\pi / C^{1 / 2}, 2\right)$. The case $p(z) \equiv C$ shows that this application is sharp (see [6, Corollary]).

7. $n$-valent functions. We shall now apply the theorems on the Euclidean distance to obtain an upper bound $n$ for the number of zeros of any solution $y(z)$ of $(1.1)$ in the unit circle. This will be equivalent to ensuring that the corresponding meromorphic functions of the restricted class are at most $n$-valent in the unit circle.

Our theorems gave us a lower bound $d$ for the distance between two zeros of any solution $y(z)$. We are therefore led to consider the following-purely geometrical-magnitude:

$$
d_{n}=1 . \text { u. b. }\left(\underset{\mu \neq v, \mu, \nu=1 \cdots n}{\operatorname{Min}}\left|z_{\mu}-z_{p}\right|\right),
$$$$
n \geqq 2 \text {, }
$$

where all the points $z_{\mu}$ are in the open unit circle $E$ and where the least upper bound is taken with respect to all sets of $n$ points $z_{1}, \cdots, z_{n}$ in $E . d_{n}$ is clearly a nonincreasing function of $n$. If we know that the above-mentioned $d$ is larger than a certain $d_{n_{n}+1}$, then every solution $y(z)$ will have at most $n_{0}$ zeros in $E$.

Clearly, $d_{2}=2$, and for $3 \leqq n \leqq 6$ it is easily seen that $d_{n}$ is equal to the side of the corresponding regular polygon inscribed in $|z|=1 . d_{7}=d_{6}=1$, as can be seen by putting the seventh point into the origin. For $n \geqq 8$ the evaluation of $d_{n}$ seems to require a separate argument. for every $n$. However, for our purposes an upper bound for $d_{n}$ will suffice and it is such an upper bound (for $n \geqq 8$ ) which we shall now obtain. We first prove 
LEMma 2. For every $m \geqq 1$, there exists a covering of the unit circle by $b(m)$ circles of diameter $4 / 3 m$; here

$$
b(m)=3 m^{2}+3 m+1 .
$$

Proof. Circumscribe a regular hexagon $H$ (of side length $s_{1}=2 / 3^{1 / 2}$ ) about the unit circle. Let $L_{m}$ be the lattice of equilateral triangles of side length $s_{m}=s_{1} / m=2 / m 3^{1 / 2}$, such that the origin is a point of $L_{m}$ and such that the sides of the equilateral triangles are parallel to the sides of $H$. The number $b(m)$ of lattice points inside or on $H$ is then given by (7.2). We describe about each of these $b(m)$ points a circle of radius $s_{m} 3^{1 / 2} / 3=2 / 3 m$. These circlesgoing through the centers of the equilateral triangles-cover the unit circle.

It follows that given any set of $b(m)+1$ points in the unit circle, at least two of these points belong to the same circle of diameter $4 / 3 \mathrm{~m}$. From (7.2) we conclude that if

$$
3 m^{2}+3 m+2 \leqq n \leqq 3 m^{2}+9 m+7,
$$

$$
m \geqq 1 \text {, }
$$

then $d_{n} \leqq 4 / 3 m$. Since

$$
3 m^{2}+9 m+7<\left(m 3^{1 / 2}+7^{1 / 2}\right)^{2}, \quad m \geqq 1,
$$

we obtain, for $n$ and $m$ connected by (7.3),

$$
d_{n} \leqq \frac{4\left(m 3^{1 / 2}+7^{1 / 2}\right)}{3 m} \frac{1}{m 3^{1 / 2}+7^{1 / 2}}<\frac{4(3)^{1 / 2}}{3}\left(1+\frac{7^{1 / 2}}{m 3^{1 / 2}}\right) \frac{1}{n^{1 / 2}} .
$$

In view of $n^{1 / 2}<m 3^{1 / 2}+7^{1 / 2},(7.4)$ yields the inequality

$$
d_{n}<\frac{4(3)^{1 / 2}}{3 n^{1 / 2}}\left(1+\frac{7^{1 / 2}}{n^{1 / 2}-7^{1 / 2}}\right)
$$

or

$$
d_{n}<\frac{4(3)^{1 / 2}}{3} \frac{1}{n^{1 / 2}-7^{1 / 2}}
$$

(7.5) holds (by (7.3)) for all $n \geqq 8$. This bound should however only be applied for $n \geqq 25$, as the right-hand side of (7.5) for $n=24$ is still larger than 1 and we saw before that $d_{7}=1$.

Theorem 5 and the bound (7.5) give now

COROLlaRY 2. Let $f(z)$ be a meromorphic function of the restricted class in $|z|<1$, such that

$$
|\{f(z), z\}| \leqq \frac{2}{1-|z|^{2}} \quad \text { for } r \leqq|z|<1,0<r<1,
$$

and let $n$ be the integral part of 


$$
\left(\frac{2}{3^{1 / 2}\left(1-r^{2}\right)^{1 / 2}}+7^{1 / 2}\right)^{2} \text {. }
$$

Then $f(z)$ is at most n-valent in $|z|<1$.

Proof. By the remarks at the beginning of this section, we have to show that

$$
d>d_{n+1} \text {. }
$$

By (5.4) and (7.5) this will hold if

$$
2\left(1-r^{2}\right)^{1 / 2} \geqq \frac{4(3)^{1 / 2}}{3} \frac{1}{(n+1)^{1 / 2}-7^{1 / 2}} .
$$

However, this inequality is equivalent to

$$
n+1 \geqq\left(\frac{2}{3^{1 / 2}\left(1-r^{2}\right)^{1 / 2}}+7^{1 / 2}\right)^{2},
$$

and we have therefore proved the corollary.

Similarly we may apply (7.5) in connection with Theorem 6 or with the corollary of [6], mentioned at the end of the last section. This last case is also contained implicitly in a theorem due to Choy Tak Taam [11, Theorem 2].

\section{BIBLIOGRAPHY}

1. L. Bieberbach, Lehrbuch der Funktionentheorie, vol. 2, 2d ed., Leipzig and Berlin, 1931

2. G. H. Hardy, J. E. Littlewood, and G. Polya, Inequalities, 2d ed., Cambridge, 1952.

3. E. Hille, Non-oscillation theorems, Trans. Amer. Math. Soc. vol. 64 (1948) pp. 234-252.

4. - Remarks on a paper by Zeev Nehari, Bull. Amer. Math. Soc. vol. 55 (1949) pp. $552-553$.

5. P. Montel, Sur les fonctions localement univalentes ou multivalentes, Ann. Ecole Norm. (3) vol. 54 (1937) pp. 39-54.

6. Z. Nehari, The Schwarzian derivative and schlicht functions, Bull. Amer. Math. Soc. vol. 55 (1949) pp. 545-551.

7. - On the zeros of solutions of second-order linear differential equations, Amer. J. Math. vol. 76 (1954) pp. 689-697.

8. - Some criteria of univalence, Proc. Amer. Math. Soc. vol. 5 (1954) pp. 700-704.

9. R. Nevanlinna, Eindeutige analytische Funktionen, 2d ed., Berlin, 1953.

10. V. V. Pokornyi, On some sufficient conditions for univalence, Doklady Akademii Nauk SSSR (N.S.) vol. 79 (1951) pp. 743-746.

11. C. T. Taam, On the complex zeros of functions of Sturm-Liouville type, Pacific Journal of Mathematics vol. 3 (1953) pp. 837-843.

WASHINGTON UNIVERSITY,

ST. Louis, Mo. 